\title{
Quelle influence de la loi HPST sur les valeurs publiques? Le cas d'un Centre Hospitalier Universitaire
}

Catherine Dos Santos*, Morad Mousli et Joëlle Randriamiarana

Enseignants-chercheurs

France Business School-Campus Clermont

4, boulevard Trudaine - 63000 Clermont-Ferrand

\section{Résumé}

Cette recherche s'intéresse à l'influence de la loi HPST sur les valeurs qui sous-tendent l'hôpital public sous l'angle des missions nouvellement introduites. Elle s'appuie sur une étude qualitative de type interprétative qui a pris la forme d'une approche exploratoire auprès d'un CHU du centre de la France. Les résultats montrent que la mise en place d'une nouvelle organisation fondée sur des nouvelles instances de direction et sur l'introduction de pôles d'activités ne modifie pas la perception des acteurs sur les valeurs. Néanmoins, la dimension managériale semble influer négativement sur ces dernières en raison principalement de l'absence de compétences ou d'appétences gestionnaires pourtant nécessaires au pilotage des nouvelles missions.

(c) 2013 IDMP/Lavoisier SAS. Tous droits réservés

Mots clés : valeurs publiques, hôpital, gouvernance, missions, management.

\section{Abstract}

What effects the HPST law has had on Public Health Values? The case of a University Hospital Centre. This study is focusing on the influence of the HPST Law on the values that underlie the public hospital from the point of view of newly introduced missions. It is leaning on an interpretative-qualitative analysis that takes the form of an exploratory approach of a CHU in the center of France. The results show that the setting up of a new organization based on a new management authority and on the introduction of new activity centers doesn't change the value perception of the actors. However, it seems that the managerial dimension has a negative impact on these values because of the 
absence of whether competencies or managerial appetencies which are necessary when running new missions.

Keywords: Public values, Hospital, Governance, Missions, Management.

\section{Introduction}

La crise hospitalière serait à attribuer en majeure partie à l'insuffisance du management des établissements en position de faiblesse face à la communauté médicale et au conseil d'administration. Elle trouverait sa résolution dans l'avènement d'un exécutif fort, programmé par l'injonction présidentielle « un seul patron à l'hôpital ». L'établissement hospitalier doit s'organiser sur le modèle de la libre entreprise, celle du monde réel où la concurrence règne et où la productivité, synonyme d'effort, est la valeur première. Concentration, gain de performances et efficience doivent se traduire par des fusions, des rapprochements, des complémentarités entre établissements. C'est dans ce contexte que la loi Hôpital, patients, santé et territoire (HPST) a réformé la notion de service public dont la crise est dénoncée depuis plusieurs années. En effet, l'établissement public n'est plus le véhicule normal du service public mais l'un des acteurs du service public en concurrence avec les autres offreurs privés de soins. Par ailleurs, la loi HPST continue de décloisonner le monde de la santé : les établissements de santé n'étant que l'une des formes particulières de prise en charge du service public de santé.

Cette révolution qui suscite de très nombreuses réactions légitimes dans le public comme dans le privé, sans compter les réactions politiques ou syndicales, appelle à l'évidence de nouveaux éclairages, notamment à l'heure où la Ministre de la Santé, Marisol Touraine, indique vouloir prendre ses distances avec la loi HPST. « La loi HPST avait balayé toutes les dispositions relatives au service public hospitalier, provoquant ainsi dans l'ensemble de la communauté hospitalière le sentiment légitime qu'il y avait là une atteinte sans précédent à l'encontre des valeurs fondatrices du service public » (propos de Marisol Touraine, septembre 2012). La problématique de cette contribution pourrait se résumer de la façon suivante : la loi HPST, en modifiant les missions de l'hôpital public, ne menace-t-elle pas les valeurs qui le sous-tendent?

Pour y répondre, notre article s'appuiera sur l'analyse d'un Centre Hospitalier Universitaire (CHU) du centre de la France et de la mise en œuvre des nouvelles missions au travers de son plan d'établissement. Cette étude de cas, de type exploratoire, a été conduite entre septembre et octobre 2012 à partir d'une méthodologie qualitative fondée sur des entretiens à visée interprétative (encadrés 1 et 2). La première partie aborde la problématique de la recherche, la seconde propose une analyse de cas et une discussion des résultats. 


\section{Encadré 1 : Présentation du cas}

L'objectif de cette étude empirique, conduite en septembre et octobre 2012, est la description et l'analyse de la réorganisation du CHU autour de nouveaux organes de décision dans le but de mettre en œuvre une gouvernance idoine à l'exercice des nouvelles missions. La présentation de l'étude comprend deux sections. La première posera le cadre des travaux réalisés à partir d'une justification du cas ainsi que d'une présentation générale du CHU. La seconde exposera la méthodologie déployée pour récolter les différents matériaux et analyser les résultantes des nouvelles missions sur les valeurs publiques.

Le CHU étudié, situé au centre de la France, est avant tout un établissement de proximité accueillant des patients qui viennent de son agglomération à hauteur de $63 \%$. C'est aussi un établissement régional puisque $19 \%$ des patients hospitalisés viennent des autres départements de la région, tandis que $9 \%$ sont originaires d'autres régions.

Le CHU est organisé en 21 pôles (17 pôles cliniques et 4 pôles médicaux-techniques) répartis sur trois sites. L'organisation par pôle a été mise en place fin 2006, suivant un certain nombre de règles :

- les responsables de pôles doivent élaborer un projet en cohérence avec le projet d'établissement;

- des objectifs d'activité et de qualité sont établis dans le cadre d'un contrat, avec en contrepartie une définition des moyens négociés;

- une politique d'intéressement aux résultats du pôle est mise en place;

- la logique de délégation de gestion fait partie des règlements intérieurs des pôles. Le CHU est composé de trois entités d'une capacité d'accueil de 1996 lits répartis de la manière suivante :

- 795 lits sur le site $n^{\circ} 1$, dont 24 postes de dialyse;

- 558 lits sur le site $n^{\circ} 2$, dont 7 postes de dialyse et d'aphérèse pédiatrique;

- 355 lits, sur le site ${ }^{\circ}$ 3, de médecine, moyen, long séjour et EHPAD dont 7 places de soins de suite et de réadaptation (SSR).

Les principales disciplines sont les suivantes : médecine, chirurgie, gynéco-obstétrique, psychiatrie, SSR, EHPAD et long séjour. La discipline médecine est la plus importante en termes de nombre de séjours en hospitalisation complète, suivie de la chirurgie et de la gynéco-obstétrique. 


\section{Encadré 2 : Méthodologie de la recherche}

Comme le thème des implications de la loi HPST sur les missions de l'hôpital public par le prisme des valeurs a été peu abordé dans les travaux antérieurs, nous avons fait le choix d'une démarche exploratoire. Elle a pris la forme d'une recherche-intervention réalisée dans le cadre d'une convention signée avec le CHU en septembre 2012, et dont les ressorts concernent la qualité du management stratégique et opérationnel au regard des dispositifs de pilotage et de contrôle de la performance. Le but de cette démarche qualitative était d'observer en profondeur la façon dont les acteurs de l'organisation perçoivent et vivent les changements inhérents à HPST.

Le cadre méthodologique de Savall et Zardet $(1975,1992,2005)$ a été retenu afin d'établir les relations entre management et performance, comportements et structures et enfin dimension économique et dimension sociale. Cette approche, de type socioéconomique nous semble être une assise idoine à nos investigations en raison, d'une part, de notre positionnement théorique interprétativiste et, d'autre part, des étapes qu'elles suggèrent. En effet, cette méthode d'intervention, nommée usuellement le processus d'amélioration (Savall et Zardet, 2000), comprend quatre étapes fondamentales : le diagnostic, le projet, la mise en place du projet et l'évaluation des résultats.

La phase de diagnostic vise précisément à mettre en évidence les dysfonctionnements potentiels et leurs coûts dans une entreprise ou une organisation à partir de trois catégories de matériaux :

- Les entretiens semi-directifs : dans notre guide d'entretien, les questions s'articulent autour de trois thèmes principaux. Sur le premier thème des missions de l'hôpital public en France, les questions concernent les grandes missions de l'hôpital, leur évolution ces dernières années ainsi que les principales modifications apportées au service public hospitalier sur le plan des soins, de la gestion, de l'image. Le deuxième thème des valeurs de l'hôpital traite les questions des valeurs sous-tendues par les missions du service public, la façon dont elles sont exprimées aujourd'hui, si elles ont motivé les interviewés dans leur choix de l'organisation. Enfin, dans le troisième thème sur les réformes hospitalières et les valeurs, les questions ont trait aux préoccupations économiques de plus en plus présentes à l'hôpital, la modification des valeurs induite par les réformes ainsi que l'impact de ces dernières sur le métier au quotidien.

- L'analyse de documents s'est focalisée sur le projet d'établissement du CHU de la période de 2010-2014 qui présente d'une part le projet médical dans un contexte réglementaire et concurrentiel et, d'autre part, les différentes missions du CHU ainsi que son nouveau mode d'organisation par pôle.

- Les rencontres avec les interlocuteurs ont permis d'avoir une compréhension du fonctionnement du CHU, le partage d'expérience de ceux qui sont sur le terrain étant une source d'information incontournable.

La première étape de notre diagnostic était donc consacrée spécifiquement à l'observation des impacts de la loi HPST sur les missions et, par suite, les valeurs du CHU. La population sondée est constituée des trois corps, soignant, non soignant et médical. Pour chaque catégorie, deux personnes ont été interviewées. Lors de l'exploitation, il a été procédé à des boucles d'abduction entre la théorie et le terrain. Des critères 
ont été respectés : l'objectivité, au travers d'une analyse croisée entre les auteurs; la validité du construit grâce à une lecture extérieure de nos propositions; la validité interne, en cherchant des points communs entre les différents éléments de réponse des acteurs sondés, et la validité externe au travers de leur portée dans le but d'en tirer des enseignements généralisables applicables à d'autres cas.

\section{La relation problématique entre les nouvelles missions de l'hôpital et les valeurs publiques}

Les deux réformes ayant fait entrer l'hôpital dans le XXI' siècle, les ordonnances de 2003 et 2005 et la loi HPST, modifient en profondeur la structure même du système hospitalier français en supprimant le service public hospitalier et en ouvrant la possibilité de confier des missions de service public à tout « offreur de soin ». D'apparence, cela peut constituer une rupture avec la place et le rôle confiés depuis des siècles à l'hôpital dans la cité et une rupture encore plus nette avec les valeurs qui le sous-tendent. La revue de la littérature abordera cette problématique en trois sections. La première aborde la question des valeurs comme socle du management et du positionnement de la culture d'entreprise. La seconde traite en particulier le contexte de la recherche au travers de la loi HPST et de ses spécificités. La dernière section propose une analyse de cette réforme sous le regard croisé des missions et des valeurs publiques.

\subsection{Cadre théorique de la recherche : les valeurs, outil de positionnement et de légitimité managérial}

La question des valeurs pose en réalité plusieurs problèmes liés aussi bien au biais managérial qu'elles constituent qu'à leur impact sur le positionnement de l'organisation dans un environnement donné. En effet, l'ensemble des valeurs partagées par des individus au sein d'un groupe (Deshanpande et Webster, 1989) constitue la culture d'entreprise que Selznick (1957) définit comme « un ensemble de représentations, symboles, valeurs, croyances et règles vécues en commun par les membres du groupe, permettant une certaine convergence des intérêts des différentes parties, et facilitant l'évolution harmonieuse du groupe social vers ses objectifs, c'est-à-dire son travail d'adaptation interne et externe ». Cette acception souligne tout particulièrement la dimension managériale des valeurs au sein d'une organisation compte tenu des impacts qu'elles peuvent avoir sur le comportement des individus et, par suite, leur performance. Elle montre également que la culture d'entreprise revêt deux dimensions distinctes, l'une interne et l'autre externe. Pour Pierre Ghewy (2006), la première vise à intégrer l'ensemble des comportements et usages jugés acceptables et nécessaires afin d'atteindre au mieux les objectifs fixés. La seconde concerne le positionnement que l'organisation souhaite avoir vis-à-vis de ses concurrents afin d'affirmer sa différence. En cela, il est possible d'envisager les valeurs de l'entreprise comme un vecteur de performance fondé sur des principes théoriques et pratiques visant à améliorer son fonctionnement et sa différenciation. C'est précisément pour cela que Thévenet (1986) les présente comme des références pour les choix stratégiques ou managériaux qui permettent aux acteurs d'avoir et de suivre un fil conducteur dans leurs actions propres. 
Dès lors, la problématique du management par les valeurs se pose dans des contextes instables de changements organisationnels forts où les missions deviennent simultanément la source et la finalité de l'ensemble des comportements des acteurs. En particulier dans le cas des organisations publiques comme les hôpitaux dont l'ADN repose davantage encore sur des normes et un positionnement distincts.

\subsection{Contextualisation de l'étude : Ia loi HPST, une ambition nécessaire pour préserver le système de santé}

C'est dans un contexte économique très sévère que sont prises, entre 2003 et 2009, les mesures qui amorcent le grand virage pour l'avenir du service public hospitalier : la réforme du financement et son application libérale, le partenariat public-privé et la suppression du service public hospitalier (Couty, 2010). Les établissements publics et privés sont confrontés à de nouveaux défis : d'abord, celui du financement par une réforme qui introduit, depuis 2004, une «tarification à l'activité » (T2A) qui permet de mieux comparer les coûts d'hospitalisation entre les deux secteurs, public et privé, mais qui peut également attiser la concurrence entre établissements de santé; ensuite, de nouvelles règles de planification hospitalière et sanitaire, prises en 2003, conduisent à répartir des parts de marché, entre les établissements, sur un territoire de santé de proximité. En ce qui concerne plus particulièrement les établissements publics, leur désenchantement est révélateur d'une crise identitaire profonde. Ils se restructurent et mettent en place une nouvelle gouvernance d'abord dans le cadre du « Plan Hôpital 2007 », traduit sur le plan législatif par une ordonnance du 2 mai 2005, ensuite dans celui du « Plan Hôpital 2012 ».

En 2005, la réflexion au sujet du futur « Plan Hôpital 2007 » entreprend une première réforme de la gouvernance hospitalière et cherche à mettre en place une coordination entre les médecins et les responsables administratifs. L'ordonnance du 2 mai 2005 constitue le volet « Gouvernance » du Plan hôpital 2007. Elle entend lever les freins qui pèsent sur les hôpitaux et mettre fin au dangereux désenchantement des praticiens et de l'ensemble du personnel soignant et administratif. L'ordonnance recentre le conseil d'administration sur ses missions stratégiques d'évaluation et de contrôle et crée un conseil exécutif composé de médecins et d'administratifs. Elle met également en place une organisation médicale interne simplifiée avec les « pôles d'activités » ou «pôles de soins » qui doivent permettre le décloisonnement de l'hôpital. Les pôles sont dirigés par un médecin, assisté d'un cadre de santé et d'un responsable administratif. Le médecin chef de pôle reçoit une délégation de décision et de gestion qu'il exerce dans le cadre d'un contrat d'objectifs.

En 2008, une étude portant sur la mise en œuvre de la réforme 2005 au sein des hôpitaux dresse un bilan plutôt favorable (Barat et al., 2008). La création du conseil exécutif apparaît comme le point le plus positif de la réforme. Les professionnels s'accordent pour mettre en avant le décloisonnement entre le corps médical et la direction ainsi que l'émergence d'une véritable implication des médecins membres dans le pilotage stratégique de l'établissement. Pourtant, dès octobre 2007, le président de la République confie à Gérard Larcher le soin de mener une concertation sur les missions de l'hôpital en indiquant son souhait de voir renforcée la responsabilité des directeurs d'hôpitaux. Le rapport, remis en avril 2008, présente une série de recommandations sur l'insertion de l'hôpital dans le parcours de soins, sur l'organisation territoriale de l'offre hospitalière et enfin sur l'organisation interne des hôpitaux (Larcher et al., 2008). En matière de gouvernance, le rapport 
propose notamment de faire évoluer les règles de l'hôpital public afin d'améliorer son pilotage (transformer le conseil d'administration en conseil de surveillance et diversifier sa composition, resserrer le conseil exécutif et le transformer en directoire), de dynamiser la gestion des directeurs d'hôpitaux et de moderniser le statut de l'hôpital public (liberté d'organisation, assouplissement des procédures).

Ầ la suite du rapport Larcher, le projet de loi HPST est présenté au Conseil des ministres du 22 octobre 2008. En matière d'organisation interne, le texte entend préciser les missions des hôpitaux et revoir les règles de gouvernance.

\subsection{Problématique de la recherche : les conditions d'exercice des missions de service public de l'hôpital aujourd'hui sont-elles de nature à modifier les valeurs?}

«La mission est comme un phare qui guide un bateau vers le port en indiquant seulement au capitaine et aux membres de l'équipage la direction à suivre » (Côté et al., 2002 : 43). Cette situation place notre revue de la littérature au niveau des objectifs fixés par la gouvernance mais également sur la façon de conduire l'ensemble des acteurs à y contribuer pleinement comme le soulignent Atamer et Calori (2003 : 373) lorsqu'ils écrivent que "l'annonce de la mission de l'entreprise stimule et oriente l'action collective de façon synthétique ». De nombreux auteurs (Campbell et al., 2001 ; Analoui, 2002; David et David, 2003; Sufi et Lyons, 2003 ; Davis et al., 2007) s'accordent à attribuer un rôle aux déclarations de mission pour motiver les employés et améliorer le leadership ainsi que pour mieux s'organiser et décider en vue d'une meilleure performance. Dès lors, les missions introduites par les réformes du système de santé renvoient, d'une part, à la nécessité de les communiquer aux acteurs des établissements publics et, d'autre part, à celle de mettre en place des structures susceptibles de favoriser leur mise en œuvre. Or, la loi HPST a supprimé toute référence à l'existence d'un service public hospitalier en définissant quatorze missions de service public, en supprimant le régime spécifique applicable aux établissements privés non lucratifs participant au service public hospitalier et en élargissant aux établissements privés lucratifs la possibilité d'exercer tout ou partie des nouvelles missions de service public.

Cette clarification des missions de service public assurées par les établissements de santé trouve une double justification. En premier lieu, l'examen des conditions d'accès aux soins montre que, sur certains territoires, aucun établissement n'assure les missions de service tandis que dans d'autres l'exercice de ces missions est parfois redondant. Il importe donc de définir précisément ces missions de service public pour que les autorités sanitaires puissent mieux les répartir. Ensuite, quand sur un territoire donné des structures privées sont disponibles pour garantir des besoins définis, il n'est effectivement pas illégitime que le décideur public choisisse d'y faire appel sachant que certains établissements de santé privés assurent déjà des missions de service public (Budet et al., 2008).

Les missions de service public s'appuient sur un certain nombre de valeurs propres à l'hôpital public, qui plongent leurs racines dans la longue histoire de l'institution hospitalière. Traditionnellement, les valeurs hospitalières sont celles du dévouement, de la vocation, de l'esprit de sacrifice. À ces valeurs d'origine religieuse s'est substituée la valeur plus laïque de solidarité, à mesure que l'hospice se transformait en hôpital (Caillol et al. 2010). Nous retrouvons cette dimension de croyance dans les travaux de Bart et Baetz (1998) 
pour lesquels le but, la raison d'être et les valeurs composent la mission. Ils définissent les valeurs d'une organisation comme "l'ensemble des croyances qui représentent les pensées et les opinions de l'entreprise sur elle-même ». Sans entonner un discours « sacerdotal », le principe fondamental des valeurs reste probablement la solidarité dans une société moderne car il incarne bien la déontologie médicale. L'égalité devant les soins, la continuité de l'offre, quel que soit le statut social, l'âge, et bien sûr, la pathologie des patients, sont des fondamentaux régaliens d'une communauté liée par une culture des valeurs plutôt que par une alliance d'intérêts (Schnapper, 1991).

Ces valeurs sont inscrites dans le préambule de la Constitution, toujours en vigueur, lequel reconnaît à tout individu le droit à la protection de sa santé. Le premier droit de la personne malade est de pouvoir accéder aux soins que son état nécessite, quels que soient ses revenus. Dès 1945, le système de protection sociale garantit aux usagers un égal et libre accès aux soins. Tous les acteurs de santé - les professionnels, les établissements et réseaux de santé, les organismes de prévention ou de soins, les autorités sanitaires doivent le mettre en œuvre au bénéfice de toute personne (Molinie et al., 2005). Ce qui fait concrètement l'honneur de l'hôpital public est l'accueil de toutes les personnes en détresse quelles qu'elles soient, puis leur prise en charge médicale et/ou psychologique, et enfin leur orientation éventuelle vers des structures de soins plus appropriées. Les différents représentants des patients reconnaissent à la fois le rôle d'accueil social de l'hôpital public et de maintien, jusqu'à présent, d'un très bon état de santé général de nos concitoyens. Lieu de soins, l'hôpital public joue donc bien aussi un rôle de lien social, fondé sur la valeur très ancienne de la solidarité, se référant à la charité qui fut la valeur fondatrice du soin. L'exercice de cette valeur demande de façon implicite de faire appel, de la part des professionnels hospitaliers tant administratifs que soignants, à des qualités humaines telles que l'accueil de la personne dans sa globalité, la courtoisie, l'écoute, le dialogue, la confidentialité et, de façon plus générale, l'estime de l'autre et le respect de sa dignité.

Dès lors, le rôle et la place donnés aux personnels hospitaliers semblent fondamentaux. La majorité des travaux (Bart et Baetz, 1998; Bart et Tabatone, 1999; Analoui et Karami, 2002) établit l'existence d'un lien entre la mission (et donc les valeurs) et la performance. Et dans ce cadre, la relation positive entre l'inclusion des employés dans le contenu des missions et la performance semble faire l'objet d'un consensus. En cas de méconnaissance, la mission ne peut pas améliorer la performance. Ainsi, Desmidt et Heene (2007) évaluent la perception par les managers et les non-managers de la déclaration de mission d'un hôpital belge. Ils mettent en évidence un écart de perception entre les deux groupes. Les managers ont une attitude plus positive à l'égard de la déclaration de mission, ce qui laisse à penser une meilleure perception des valeurs. L'influence sur la performance dépend donc du processus de développement et de mise en œuvre de la déclaration de mission (Bart et Tabatone, 2000). Mais depuis quelques années, et également l'instauration de la loi HPST, les personnels hospitaliers manifestent leur inquiétude concernant un risque important de modification du système des valeurs auquel est adossée la pratique de soins. Jusqu'à présent, les préoccupations d'ordre économique, omniprésentes à l'hôpital ne venaient qu'en second rang, après les valeurs de soins et de dévouement désintéressé.

La suppression du service public hospitalier et l'avènement d'une gouvernance pour un hôpital-entreprise ne sont-ils pas les signaux d'une rupture avec les valeurs qui fondent depuis très longtemps les missions de l'hôpital public et qui donnent sens à l'action de ses 
personnels? L'analyse interprétative du processus mis en œuvre par un CHU dans le cadre de son plan d'établissement pourrait nous aider à comprendre l'hypothétique relation entre missions et valeurs.

\section{Les missions du CHU ou le passage des valeurs publiques aux valeurs d'efficience}

Afin d'analyser au mieux les éventuels impacts d'HPST sur les valeurs du CHU, il est nécessaire d'aborder aussi bien les conséquences organisationnelles que la façon dont les nouvelles missions modifient le regard que les acteurs portent sur cette situation. Pour ce faire, les deux premières sections proposeront la restitution des résultats suivant le cadre de Savall et Zardet avec, d'une part, notre jugement tiré des observations directes et de l'analyse de documents et, d'autre part, la perception des personnes interviewées sur la relation missions-valeurs autour de verbatim tirés des entretiens. La troisième présentera un prolongement de l'ensemble des résultats en forme de bouclage théorique dans le but de répondre à notre question de recherche.

\subsection{Ce que nous révèle la nouvelle organisation du $\mathrm{CHU}$ du point de vue de ses missions}

La mission générale du CHU demeure inchangée puisqu'il s'agit toujours « d'assurer les examens de diagnostic, la surveillance et le traitement des malades, des blessés et des femmes enceintes ». En revanche, l'introduction des nouvelles missions ayant trait à la politique territoriale de santé, à la coordination des soins ou encore à la garantie de sécurité sanitaire justifie la répartition des activités et responsabilités dans le «projet d'établissement $»$. Ce projet est composé de trois dimensions clés que nous avons identifiées comme étant le projet médical, le projet social et le projet de gestion.

Le projet médical définit les orientations de la politique médiale pour 5 ans (20102014) et répond en priorité aux missions de coordination ainsi qu'à la permanence des soins et d'enseignement et recherche tout en s'inscrivant dans la continuité du projet préalable (2006 - 2009). Il s'inscrit aussi dans le cadre des orientations nouvelles de la politique de santé telles que définies par les plans de santé publique. Cela signifie, en outre, que la mission confiée au CHU en matière de coordination des soins et de l'offre est fondée sur une meilleure coopération avec les autres parties prenantes du système de santé, en particulier les médecins de villes et les cliniques. C'est pour cette raison que le CHU propose une offre de soins de proximité qui s'intègre dans le périmètre du territoire de santé tel qu'il est défini par le SROS, c'est-à-dire qui promeut une réponse adaptée à la région.

L'autre grand pendant de ce projet médical reste la recherche universitaire propre au CHU mais également fortement souhaitée par la réforme. En effet, le projet rappelle clairement que «la dimension universitaire de notre mission de CHU doit d'une certaine manière être sanctuarisée pour ne pas être sacrifiée dans une vision à court terme strictement comptable ». Sur le plan de l'enseignement et de la formation, on note une forte présence d'internes en médecine (442) dont 275 sont rémunérés directement par le CHU. À l'enseignement traditionnel s'ajoute la formation desdits internes dans différents domaines (lutte contre les infections, prévention des accidents d'exposition du sang, etc.) destinée à améliorer leur performance en qualité d'acteur du système de soins, mission ô combien ciblée par la loi HPST. 
Le projet social répond à la nécessité de mieux gérer les ressources humaines dans le cadre d'une politique de retour à l'équilibre budgétaire qui implique une reconsidération de sa gestion sociale autour de quatre interrogations-clés :

- Comment maintenir et renforcer parmi les personnels le sentiment d'appartenance à une communauté professionnelle au-delà de la diversité des métiers et statuts?

- Comment permettre à chacun de construire un projet personnel dans un ensemble si vaste et si complexe?

- Comment rendre les efforts demandés à chacun profitables pour l'ensemble de la communauté ? Comment enfin donner un sens et une lisibilité pour l'extérieur à notre gestion des ressources humaines?

Afin d'y répondre, le projet social a été construit dans le prolongement des résultats obtenus entre 2006 et 2009 autour de deux fils conducteurs : a) la valorisation des ressources humaines qui passe par des actions précises liées aussi bien à la gestion des personnels qu'aux pratiques hospitalières en matière de travail; b) les conditions de vie au travail et la gestion des préoccupations individuelles. En effet, une politique de gestion du climat social, d'accueil et d'accompagnement des personnels nouvellement recrutés ou encore de préventions de risques psychosociaux a été mise en œuvre toujours dans le but de mieux remplir la mission de développement continu des individus. En outre, le projet social est avant tout un support au dialogue social dans l'établissement. Il trace des perspectives, propose des objectifs, suggère des méthodes et des moyens tout en rappelant la nécessité d'une bonne gestion des ressources.

Les actions inscrites au Projet de gestion 2010-2014 répondent à cet objectif sur un plan financier et à la volonté de mettre en œuvre des procédures et des outils de gestion adaptés aux dispositions de la loi HPST et de ses décrets d'application notamment pour ce qui concerne la contractualisation interne. Il s'ensuit que les missions assurées directement ou indirectement par les parties prenantes de ce projet sont particulièrement nombreuses, fussent-elles d'ordre médical ou social. Ceci implique que le rôle du conseil de surveillance est central dans la mise en œuvre des différents objectifs assignés et que le projet de gestion s'articule autour de la nécessité de mettre en place une gouvernance, un management et des outils de gestion ad hoc. L'introduction d'outils et dispositifs de gestion spécifiques ainsi que d'un système d'information mieux adapté aux exigences de clarté et d'équilibre économique révèlent, en effet, l'orientation du CHU vers une organisation et une gestion éloignées des préoccupations de sa mission originelle.

En somme, cette analyse de la restructuration du CHU et des nouvelles missions inscrites dans son projet d'établissement (projet médical, projet social, projet de gestion) montre que les objectifs assignés par la loi HPST ont modifié trois dimensions de la gestion du CHU : la gouvernance hospitalière fondée sur la collaboration des parties prenantes régionales à l'exercice de l'ensemble des missions; le périmètre des nouvelles instances de décision et leurs relations fondés sur la nécessité d'une gestion structurée et efficace des ressources humaines, matérielles, techniques et financières; les missions nouvellement introduites qui bouleversent les usages et les pratiques au sein de l'établissement de telle sorte que la dimension managériale, voire gestionnaire, semble être le moteur de l'activité. Cela pose en creux la question de la place du management dans la conduite des nouvelles missions évaluées à l'aune des valeurs publiques et l'hypothèse d'un intervalle marqué entre elles en raison précisément des compétences managériales mal ou peu introduites. 
C'est à cette fin que nous proposions un prolongement de nos investigations au travers du regard d'acteurs du CHU sur les ressorts et conséquences de ces missions et de la façon dont elles sont «managées » sur les valeurs publiques.

\subsection{Perception des acteurs au sujet de l'impact des nouvelles missions sur les valeurs publiques}

L'introduction de nouvelles missions au CHU étudié ont-elles modifié la perception des acteurs sur les valeurs qui sous-tendent leur activité? Le mode de gouvernance fondé sur la relation entre trois entités fortes - Directoire, Conseil médical d'établissement (CME), Conseil de surveillance (CS) - a amorcé des changements notoires sur la perception des missions originelles du CHU mais également la façon dont elles s'exercent au quotidien.

Les missions introduites par la réforme HPST ont été vécues par certains acteurs du CHU comme une forme de dichotomie en raison, d'une part, des missions traditionnelles et, d'autre part, de la difficulté de les exercer dans le cas d'une organisation publique, en particulier en santé. Un cadre infirmier indiquait ainsi son scepticisme quant à l'avènement de missions nouvelles plus tournées vers la dimension économique : «L'hôpital entreprise est un concept en vogue, il suggère un questionnement : peut-on gérer un hôpital comme une entreprise? ». Cette interrogation renvoie en partie à la difficulté d'une telle réforme d'amorcer des changements organisationnels forts comme le contrôle de gestion, la prise en compte de la performance des services sur un plan budgétaire dans une optique d'équilibre budgétaire nommée parfois « rentabilité ».

Cette vision très en distance avec l'esprit des missions nouvelles est globalement partagée pour ce qui est de la finalité majeure de la réforme, à savoir la modification de la gouvernance et de l'organisation à des fins de gestion améliorée comme le souligne un professeur de médecine : «Développement non maîtrisé d'une culture médico-soignante de la qualité, de la rentabilité et de la concurrence ». Ce dernier insiste pourtant sur "l'intérêt de mieux sensibiliser les acteurs de l'hôpital aux contraintes du marché de santé ». Ce qui traduit la prise de conscience d'une nécessaire modification du CHU au regard de son environnement (médecins privés, cliniques, ARS) et d'un contexte économique très instable et incertain. Dès lors, les missions originelles revêtent une dimension quasi salutaire puisqu'elles s'inscrivent dans une démarche non mercantile et fondée avant toute chose sur les soins. «Soins de proximité, soins aux indigents, soins de référence, enseignement, recherche académique clinique et translationnelle », explique un membre de la CME lorsqu'il s'agit de décrire les grandes missions premières de l'hôpital public. Une telle définition souligne, en outre, la prévalence de la dimension sanitaire sur toutes les autres considérations, fussent-elles économiques ou financières.

Cette dimension est-elle corrélée aux valeurs publiques traditionnellement attribuées aux hôpitaux publics? Cela semble être le cas pour au moins deux raisons. D'une part, les valeurs publiques citées par les différentes personnes interrogées - «égalité, gratuité, solidarité » - renvoient inéluctablement à la nécessité de soigner les «indigents »sans considération autre que celle de la qualité du soin. D'autre part, le service public, tel qu'il a été conçu, se prévaut d'une gratuité des soins de telle sorte que l'introduction d'une tarification nouvelle ne fait que renforcer la dichotomie entre la mission originelle et les nouvelles. En effet, la dimension financière particulièrement renforcée par la loi et, au 
CHU, par le projet de gestion, semble constituer plus qu'un paradoxe, un frein aux valeurs publiques. Un professeur de médecine souligne que «l'irruption du tout financier induit un comportement de technicien chez les médecins avec un éloignement important par rapport au patient (approche contractuelle) et par rapport à la structure et une approche comptable du temps de présence et de la rémunération ». Ce verbatim montre que la perception des changements opérés au sein du CHU ne plaide pas nécessairement en faveur d'un alignement du comportement des acteurs sur les orientations stratégiques posées par HPST.

Deux pendants peuvent asseoir cet argument. Tout d'abord, celui d'ordre structurel. Considérant que les structures nouvellement établies pour permettre une meilleure mise en œuvre des orientations stratégiques du CHU ne sont pas acceptées ou comprises par les acteurs, elles peuvent entraîner des dysfonctionnements à différents niveaux de l'organisation. Par exemple, les nouvelles entités de gouvernance sont perçues par certains acteurs comme impertinentes comme l'exprime un cadre de santé : "Nous assistions à un transfert de responsabilités vers l'administration avec une concentration des pouvoirs directifs et décisionnels ». Rejoint sur ce point par un professeur de médecine pour lequel on observe « une atomisation des structures regroupées en conglomérats non fonctionnels ». L'autre pendant, d'ordre managérial et corrélé au premier, renvoie à la nécessaire contribution du tout un chacun dans la réussite du projet. En effet, les différents projets déployés depuis 2010 au sein du CHU ne semblent pas recevoir une réelle et totale adhésion des individus, certes sensibilisés à la nécessité d'améliorer la gestion hospitalière, mais pas convaincus par les actions entreprises. Un membre de la CME constate ainsi que les modifications apportées tant sur les structures que les modes de management entraînent un "désinvestissement des acteurs hospitaliers par rapport à leur mission de soins et leurs missions fonctionnelles ». Constat d'autant plus critique qu'il contribue à éloigner le CHU des valeurs sous-tendues jusqu'alors et que plusieurs acteurs sondés insistent sur le fait que ces dernières constituent la principale raison de leur engagement.

En cela, la relation entre les missions du CHU, la façon de les conduire sur un plan organisationnel et managérial et les valeurs publiques devient la pierre d'achoppement du débat qui porte sur la nécessité de changer l'hôpital public. Allons plus loin sur cette relation qui a suggéré de nombreuses interrogations au sein du personnel du CHU, en particulier celle qui concerne la façon de le gouverner. «L'hôpital n'a rien de libéral » affirme un cadre de santé, insistant sur l'historique des hôpitaux publics et sur les amalgames à éviter lorsque l'on aborde l'épineux problème de rentabilité de ces derniers. En effet, la nécessité d'un équilibre financier ne doit pas être confondue avec l'obligation de résultat des entreprises privées bien que parfaitement légitime pour mener à bien les activités sanitaires et sociales. Un professeur de médecine rappelle ainsi que «l'introduction du financier dans la démarche de soins est indispensable pour assurer la meilleure répartition des moyens entre les citoyens ». Argument qui montre que la dichotomie entre les valeurs prônées historiquement par le CHU et celles introduites implicitement par la réforme HPST n'est pas si profonde. Un membre du CS souligne ainsi que «les valeurs dépendent des hommes et non des systèmes », insistant sur la nécessité de fonder le changement sur les hommes et, par conséquent, sur la qualité du management. Plusieurs individus semblent partager cette perception sur l'introduction de valeurs d'efficience plus idoines pour fédérer l'ensemble des acteurs et porter le CHU dans ses nouvelles orientations stratégiques, à l'instar de ce membre du CS pour lequel «les valeurs soignantes sont, il me semble, indépendantes de 
la situation économique. Parfois, au niveau de l'encadrement, nous pouvons être amenés à nous questionner sur nos valeurs de gestionnaire lorsque nous proposons des moyens humains en inadéquation avec l'activité ».

Cette analyse interprétative fondée sur la perception de plusieurs personnes sondées durant notre étude nous ramène donc à notre hypothèse de départ selon laquelle la modification des missions du CHU est susceptible d'influencer les valeurs publiques. Les potentielles relations entre missions et valeurs semblent, en outre, devoir être évaluées sur le terrain du management.

\subsection{Les enseignements de la recherche}

La loi HPST portant réforme sur les modes de gouvernance des hôpitaux publics a eu pour effet de modifier à la fois le mode de fonctionnement de ces organisations et leurs missions, mais également la façon de les réaliser au travers du pilotage des nouvelles orientations stratégiques. Dès lors, la dimension managériale s'immisce pour devenir la clé de voûte de notre étude. C'est à la lumière de celle-ci et de la nouvelle structure mise en place que nous nous proposons de discuter les résultats de l'analyse organisationnelle et interprétative.

La problématique de gestion à laquelle sont confrontés les établissements publics de santé a amorcé les changements organisationnels et structurels auxquels nous assistons depuis près de dix ans. En effet, la réforme de la gouvernance, visant à améliorer le fonctionnement et l'exercice des missions de l'hôpital, s'est fondée sur l'introduction d'objectifs par services regroupés en pôles (Larcher et al., 2008). À l'instar des Business Units dans les modèles organisationnels anglo-saxons, ces pôles ont pour vocation de permettre à leur directeur de piloter de façon plus libre et directe les différentes activités recensées dans le projet d'établissement (Budet et al., 2008 ; Delas, 2011). Or, cette volonté de considérer les directeurs de pôles comme des managers responsables de leur «business » n'a pas été réellement vécue comme telle dans les établissements de santé, notamment dans notre cas. En effet, le pouvoir délégué aux pôles semble insuffisant pour permettre une mise en œuvre des orientations dessinées par la réforme HPST, en particulier sur un plan budgétaire.

L'une des raisons probables est la création de nouvelles instances de décision (Directoire, $\mathrm{CME}$ et CS) qui n'ont pas parfaitement redistribué les cartes et les missions. Aussi, comme le soulignait De Pouvourville (2010 : 26), «Dans le système ancien, il y avait des équilibres de pouvoir. Les médecins ayant une autonomie professionnelle et une vraie responsabilité en tant que chefs de service étaient certes statutairement sous la responsabilité hiérarchique de l'hôpital mais, d'une certaine façon, ils avaient réussi à préserver une sphère de responsabilité professionnelle ». Il indique ainsi, comme dans le cas du CHU étudié, la difficulté de déléguer réellement un pouvoir sous la contrainte budgétaire, créant de fait une tension inhérente à la frustration de ne pas obtenir ce qui vous a été promis. En cela, conclut Delas (2011 : 19) dans des travaux portant sur le rôle de l'hôpital public en qualité d'acteur territorial, que "le directeur d'aujourd'hui doit impérativement, en plus d'être un bon gestionnaire, disposer de qualités relationnelles évidentes, être doté d'un sens politique certain face aux injonctions contradictoires qu'il doit quotidiennement gérer et porter désormais une attention renouvelée aux caractéristiques socio-sanitaires ainsi qu'à la géographie du territoire que son établissement prend en charge ». 
La conclusion de Delas renvoie à la dimension managériale sur laquelle notre analyse interprétative s'est conclue, en particulier pour conduire les missions introduites en matière de gouvernance. En effet, la perception des missions sous l'angle des valeurs publiques a montré la prévalence de la dimension économique sur le soin. Elle a également mis le doigt sur la difficulté de pilotage des orientations au sein des pôles sous l'égide des nouvelles instances en raison, a priori, de l'absence de compétences managériales ad hoc, en partie chez les chefs de pôles, choisis en raison de leur légitimité médicale davantage que pour leurs appétences et compétences en gestion, ce que rappelle De Pouvourville (2010 : 26) lorsqu'il résume la situation ainsi : «On vend aux médecins une réforme en leur disant qu'ils vont pouvoir développer des stratégies nouvelles, avec des outils de gestion partiellement inadaptés, et dans un contexte de renforcement du contrôle hiérarchique descendant de la tutelle publique sur les établissements et donc sur les services médicaux. Voilà, pourquoi les médecins ont le blues ». En outre, la relation aux missions et, par suite, à leur acceptation comme à leur réalisation passe par l'exercice d'un management adapté à l'organisation du CHU, à son contexte et à son histoire. Ce qui implique que les valeurs sous-tendues ne modifient pas l'hôpital, mais lui imposent de trouver luimême les réponses aux questions posées là encore en creux par les réformes, dont la loi HPST : Comment utiliser les compétences médicales au mieux? Comment prendre les décisions les plus opportunes ? Comment parvenir à exercer l'ensemble des missions avec le concours de l'ensemble des parties prenantes internes et externes de l'hôpital public?

Nos résultats montrent, en outre, que les difficultés de mises en ouvre des missions assignées au CHU ne relèvent pas exclusivement de la nature des orientations stratégiques ou d'une structure fondée en partie sur des modèles organisationnels privés. D'ailleurs, les résultats obtenus depuis trois ans attestent des nombreuses réussites à la fois dans la mise en place des projets et dans le mode de fonctionnement. La dernière enquête gouvernementale réalisée sur la question de la réforme HPST, coordonnée par Fellinger et Boiron (2012 : 30) corrobore cet état de fait. Elle expose, en ce qui concerne la nouvelle gouvernance : "Malgré le poids des changements introduits par cette réforme, la charge de travail subséquente, et les controverses ou désaccords qu'elle suscite jusqu'à ce jour, les hôpitaux et leurs responsables ont été en mesure de mettre en auvre concrètement l'essentiel des nouvelles dispositions d'organisation, sans créer de rupture dans le fonctionnement de la mission principale de l'hôpital : accueillir et soigner des patients, former des professionnels, mener des activités de recherche ». Nos résultats révèlent davantage la nécessité d'une approche où les dimensions médicale, sociale et économique ne seraient pas traitées indépendamment les unes des autres au risque de créer des îlots sans possibilité de communication, ni de coordination.

En résumé, la réussite du projet d'établissement dans les années à venir dépend moins des instances de gouvernance que de leurs compétences managériales qui devront être transversales et partagées. En effet, leur capacité à reconstruire un ensemble de valeurs adaptées au contexte nouveau et mouvant du système de santé sera décisive dans l'implication et la performance des acteurs. Ce système de valeurs correspondra d'autant plus à l'hôpital que ces dernières seront répandues (Schein, 1990 ; Weiner, 1988) et permettront, selon Thévenet (1986), « de construire la «vision du monde » de l'entreprise, qui est une dimension première de sa culture ». Les valeurs ne sont donc pas modifiées par les missions, mais par la façon de les remplir dans un système de valeurs donné qui plus est ouvert aux parties prenantes externes (médecins privés, cliniques, ARS). 


\section{Conclusion}

Notre recherche, partant du constat que les établissements publics sanitaires et sociaux faisaient l'objet de modifications profondes de leur mode de gouvernance dans le cadre de la loi HPST et des valeurs publiques qui les sous-tendent, avait pour finalité de comprendre les relations hypothétiques entre les missions nouvellement introduites et lesdites valeurs. Les résultats obtenus dans le cadre de l'étude exploratoire de type qualitatif et interprétatif d'un CHU nous permettent de tirer les enseignements suivants.

Tout d'abord, la nouvelle organisation du CHU mise en place dans le but de mieux gouverner l'ensemble des activités répond à deux injonctions clés : la nécessité de prendre en compte les parties prenantes internes et externes de l'hôpital. En effet, la loi HPST, en introduisant une logique de territoires, a rendu nécessaire la gestion transversale et coopérative au sein du système de santé organisé alors en région sous la tutelle singulière des ARS. Ceci a eu pour effet de modifier la responsabilité du CHU en tant que partie prenante centrale de ce système et, donc, la façon de le gérer en tant que tel. C'est pour cette raison que les instances de pilotage ont été redéfinies afin, d'une part, de redistribuer les pouvoirs de façon à mieux intégrer cette dimension territoriale et, d'autre part, de mieux associer les différentes catégories de personnel du CHU. Dès lors, dans une logique de structuration en pôles d'activité d'approche organisationnelle anglo-saxonne, le CHU a entamé une nouvelle gestion fondée aussi bien sur l'ancrage territorial que sur un fonctionnement par contrats d'objectifs. Cette deuxième dimension montre la nécessité d'une prise en compte accrue de la performance, en particulier économique, dans la nouvelle gouvernance du $\mathrm{CHU}$ et de ses orientations stratégiques résultant de l'introduction de missions spécifiques. Notre étude documentaire (plan d'établissement) et nos observations révèlent que cette organisation, bien qu'ayant fait l'objet de nombreux débats, est plus idoine à la réalisation des missions nouvelles comme en témoigne la mise en œuvre du projet de gestion. Nous retrouvons, à ce titre, les conclusions du rapport d'enquête réalisé en début d'année 2012 par Fellinger et Boiron sur les retombées de la loi HPST.

Ensuite, les résultats de l'enquête par entretiens montrent que l'impact de ces nouvelles missions du CHU sur les valeurs (équité, gratuité des soins, traitement des indigents, etc.) n'est pas perçu de façon négative ou, tout du moins, pas en raison de leur nature. En effet, de nombreux acteurs interrogés ont souligné l'importance des nouvelles missions sur un plan managérial plus que sur celui des orientations ou de la nouvelle structure. Ceci semble s'expliquer pour deux raisons essentielles : d'un côté, la qualité du management fonde la réussite de tout projet de changement organisationnel fort de telle sorte que le rôle des managers devient la clé de voûte de l'exercice effectif et efficace des missions ; d'un autre côté, dans le cas particulier des hôpitaux, la dimension managériale renvoie aussi bien aux compétences fragiles en matière de gestion des nouveaux chefs de pôles qu'à leur mode de désignation. Ces derniers étant désignés souvent en raison de leur statut « médical » sans prise en compte de leur appétence pour la gestion ou compétences en management. En outre, les missions de dimension plus économique que médicale introduites par la loi HPST influencent négativement les valeurs du point de vue des acteurs en raison de compétences managériales peu ou mal introduites, notamment en matière de pilotage des objectifs dans les nouveaux pôles.

Enfin, la méthodologie déployée et le fait de n'avoir étudié qu'un seul cas constituent une limite qui ne nous permet pas de généraliser nos conclusions. Des recherches futures 
sur l'impact de l'introduction d'outils de gestion et de dispositifs de pilotage sur les compétences managériales ou le management par les valeurs pourraient apporter un éclairage supplémentaire sur la portée de ces premiers résultats.

\section{Bibliographie}

ANALOUI F., KARAMI A., (2002). CEO 's and Development of the Meaningful Mission Statement, Corporate Governance 2 (3), 13-20.

ATAMER T., CALORI R., (2003). Diagnostic et décisions stratégiques, Dunod, Paris.

BARAT S. et al., (2008). Etude qualitative relative à la mise en place de la nouvelle gouvernance dans les établissements publics de santé, EHESP, juin.

BART C.K., BAETZ M.C., (1998). The Relationship between Mission Statements and Firm Performance: an Exploratory Study, Journal of Management Studies 35 (6), 823-853.

BART C.K., TABONE J.C., (1999). Mission Statement Content and Hospital Performance in the Candian Notfor-Profit Health Care Sector, Health Care Management Review 24 (3), 18-29.

BART C.K., TABONE J.C., (2000). Mission Statement in Canadian Not-for-Profit Hospitals: Does Process Matter? Health Care Management Review 25 (2), 45-63.

BUDET J-M., PERICARD P., VIGNERON E., (2008). Territoires et santé : pour servir le débat public autour du projet de loi Hôpital, patients, santé, territoires, Revue hospitalière de France 525, 42-57.

CAILlOL M., LE COZ P., AUBRY R., BRECHAT P.H., (2010). Réformes du système de santé, contraintes économiques et valeurs éthiques, déontologiques et juridiques, Santé Publique 22 (6), 625-636.

CÔTÉ M., MALO M.C., (2002). La gestion stratégique, Gaëtan Morin, Paris.

COUTY E., (2010). Hôpital public : le grand virage, Les Tribunes de la santé 28, 39-48.

COUTY E., KOUCHNER C., LAUDE A., TABUTEAU D., (2010). La loi HPST, regards sur la réforme du système de santé, Presses de l'EHESP, Paris.

DAVID F.R., DAVID F.R., (2003). It's Time to Redraft your Mission Statement, Journal of Business Strategy Jan Feb, 11-14.

DAVIS J., RUHE J., LEE M., RAJADHYAKSHA U., (2007). Mission Possible: Do School Mission Statements Work? Journal of Business Ethics 70, 99-110.

DE POUVOURVILLE G., (2010). La crise d'identité des médecins face au nouveau management de 1'hôpital, Le journal de l'École de Paris du management 86, 22-29.

DELAS A., (2011). L'hôpital public un nouvel acteur territorial entre aménagement sanitaire et rivalités stratégiques, Hérodote 143, 89-119.

DESHPANDE R., WEBSTER F-E., (1989). Organizational culture and marketing: defining the research agenda, Journal of Marketing 53 (1), 3-15

DESMIDT S., HEENE A., (2007). Mission statement perception: Are we all on the same wavelength? A case study in a Flemish hospital, Health Care Management review, 32 (1), 77-87.

DOS SANTOS C., MOUSLIN M., (2011). Une approche de type socio-économique comme alternative à la réforme risquée des établissements hospitaliers : le cas d'un service hématologique, Revue des sciences et de gestion 83,133-152.

FELLINGER F., BOIRON F., (2012). Mission hôpital public, Ministère du Travail, de l'Emploi et de la Santé, Paris.

GHEWY P., (2006). La légitimation sociale de l'activité économique de l'entreprise par le discours sur les valeurs : le cas des TPE, Marché et organisations 2, 97-108.

LARCHER G. et al., (2008). Les missions de l'hôpital, La documentation française, Paris. 
MOLINIE E.,(2005). L'hôpital public en France : bilan et perspectives, Avis et Rapports du Conseil Economique et Social, juin, $259 \mathrm{p}$.

SAVALL H., (1975). Enrichir le travail humain dans les entreprises et les organisations, Dunod, Paris.

SAVALL H., ZARDET V., (1992). Le nouveau contrôle de gestion, méthode des coûts-performances cachées, Éditions comptables Malesherbes, Paris.

SAVALL H., ZARDET V., (2004). Recherche en Sciences de Gestion : Approche Qualimétrique, Observer l'objet complexe, Collection Recherche en Gestion, Economica, Paris.

SAVALL H., ZARDET V., BONNET M., (2000). Méthodologie qualimétrique d'évaluation des coûts cachés, Actes du Congrès International de Méthodologie, Academy Of Management, 256-282.

SELZNICK P., (1957). Leadership in administration. A Sociological Interpretation, University of California, Berkeley.

SCHEIN E. H., (1990). Organizational Culture, American Psychologist 45, 109-19.

SCHNAPPER D., (1991). La France de l'intégration, Gallimard, Paris.

SCHWEYER F-X., (2006). L'hôpital, une transformation sous contrainte Hôpital et hospitaliers dans la revue, Revue française des affaires sociales 4, 203-223.

SUFI T., LYONS H., (2003). Mission Statements Exposed, International Journal of Contemporary Hospitality Management 15, 255-262.

TABUTEAU D., (2010a). La loi HPST peut mener à la privatisation du système de santé, Pharmaceutiques juin-juillet 2010 .

TABUTEAU D., (2010b). Du plan Seguin à la loi HPST : les évolutions de la politique de santé, Les Tribunes de la santé 2010/5 HS 1,37-51.

THÉVENET M., (1986). Audit de la culture d'entreprise, Les éditions d'organisation, Paris.

VALETOUX F., (2011). La loi HPST trente mois après, Cahiers Hospitaliers 283.

VALLANCIEN G., (2008). Réflexions et propositions sur la gouvernance hospitalière et le poste de président du directoire, Ministère de la santé, de la jeunesse, des sports et de la vie associative, Paris.

WEINER Y., (1988). Forms of Value Systems: A Focus on Organizational Effectiveness and Cultural Change and Maintenance, Academy of Management Review 13, 534-45. 
